\title{
BMJ Open Implementation of the StandingTall programme to prevent falls in older people: a process evaluation protocol
}

\author{
Morag E Taylor (D) , , 2 Chris Todd (D) , 3,4,5,6 Sandra O'Rourke, ${ }^{1}$ \\ Lindy M Clemson (D) ,7,8 Jacqueline CT Close, ${ }^{1,2}$ Stephen R Lord, ${ }^{1,9}$ \\ Thomas Lung (D) , ${ }^{10,11}$ David J Berlowitz (D) , ${ }^{12,13}$ Jannette Blennerhassett, ${ }^{12}$ \\ Jessica Chow, ${ }^{1}$ Julia Dayhew, ${ }^{14}$ Helen Hawley-Hague (D) ${ }^{3,4}$ Wendy Hodge, ${ }^{15}$ \\ Kirsten Howard (D) , ${ }^{11}$ Pamela Johnson, ${ }^{16}$ Reena Lasrado, ${ }^{3,4}$ Garth Mclnerney, ${ }^{1}$ \\ Marita Merlene, ${ }^{15}$ Lillian Miles, ${ }^{1}$ Catherine M Said, ${ }^{13,17,18}$ Leanne White, ${ }^{1}$ \\ Nicola Wilson, ${ }^{19}$ Avigdor Zask, ${ }^{14}$ Kim Delbaere (D) ${ }^{1,9}$
}

To cite: Taylor ME, Todd C, O'Rourke S, et al. Implementation of the StandingTall programme to prevent falls in older people: a process evaluation protocol. BMJ Open 2021;11:e048395. doi:10.1136/ bmjopen-2020-048395

- Prepublication history for this paper is available online. To view these files, please visit the journal online (http://dx.doi org/10.1136/bmjopen-2020048395).

Received 26 December 2020 Accepted 06 July 2021

Check for updates

(C) Author(s) (or their employer(s)) 2021. Re-use permitted under CC BY-NC. No commercial re-use. See rights and permissions. Published by BMJ.

For numbered affiliations see end of article.

Correspondence to

Prof Kim Delbaere;

k.delbaere@neura.edu.au

\section{ABSTRACT}

Introduction One in three people aged 65 years and over fall each year. The health, economic and personal impact of falls will grow substantially in the coming years due to population ageing. Developing and implementing costeffective strategies to prevent falls and mobility problems among older people is therefore an urgent public health challenge. StandingTall is a low-cost, unsupervised, home-based balance exercise programme delivered through a computer or tablet. StandingTall has a simple user-interface that incorporates physical and behavioural elements designed to promote compliance. A large randomised controlled trial in 503 community-dwelling older people has shown that StandingTall is safe, has high adherence rates and is effective in improving balance and reducing falls. The current project targets a major need for older people and will address the final steps needed to scale this innovative technology for widespread use by older people across Australia and internationally.

Methods and analysis This project will endeavour to recruit 300 participants across three sites in Australia and 100 participants in the UK. The aim of the study is to evaluate the implementation of StandingTall into the community and health service settings in Australia and the UK. The nested process evaluation will use both quantitative and qualitative methods to explore uptake and acceptability of the StandingTall programme and associated resources. The primary outcome is participant adherence to the StandingTall programme over 6 months.

Ethics and dissemination Ethical approval has been obtained from the South East Sydney Local Health District Human Research Ethics Committee (HREC reference 18/288) in Australia and the North West- Greater Manchester South Research Ethics Committee (IRAS ID: 268954) in the UK. Dissemination will be via publications, conferences, newsletter articles, social media, talks to clinicians and consumers and meetings with health departments/managers.

Trial registration number ACTRN12619001329156.

\section{Strengths and limitations of this study}

- StandingTall uses technology to deliver unsupervised, progressive and tailored exercise to prevent falls in community-dwelling older people.

- Implementation of StandingTall into community and health service settings will be evaluated.

- The study will use quantitative and qualitative methods, including adherence to the intervention.

- The study will explore facilitators and barriers to uptake and adherence.

- COVID-19 caused a shift to telehealth delivery; facilitators and barriers to these procedures will be explored.

\section{INTRODUCTION}

Falls and fall-related injuries can lead to mobility-related disability and loss of independence in older people, causing both personal and community burden. ${ }^{1}$ Falls are reported as the leading cause of injury-related hospitalisation in older people and the seventhleading cause of all age disease burden in the western world. ${ }^{2}$ This will result in increased demand for health services unless effective and cost-effective fall prevention strategies can be implemented. ${ }^{3}$ By 2051, the projected total annual Australian health bill for fallrelated injury will reach $\$ A 1.4$ billion, which will create demands on the health system that will be difficult to meet. ${ }^{4}$

There is clear evidence that falls in older people can be prevented with appropriatelydesigned exercise programmes. A Cochrane review concluded that exercise interventions are among the most effective strategies to reduce fall rates in community-dwelling older people. ${ }^{5}$ Therefore, widespread implementation of exercise as a single intervention is a fall 
prevention priority. Well-designed exercise programmes, including moderate to high-intensity balance exercise of sufficiently high dose (2-3 hours per week) over periods of 6 months or more, can reduce falls by up to $39 \% .{ }^{6}$ Despite strong evidence that falls can be prevented, older people and many health professionals are often not aware that exercise is an effective strategy for preventing falls. ${ }^{1-9}$ Further, achieving a sufficient dose for fall prevention effects in clinical practice can be difficult to achieve. ${ }^{1011}$ There is a clear gap between evidence and action. ${ }^{12}$

Worldwide, national policies have developed fall prevention action plans. ${ }^{13-15}$ However, currently there is limited access to fall prevention programmes and therefore an unmet need for delivering accessible, evidence-based fall prevention interventions to older people. ${ }^{16-18}$ Major shortcomings of many current programmes are that they are offered relatively short-term and lack long-term sustainability. ${ }^{171920}$ Therefore, these programmes do not offer long-term protection from falls and/or functional decline. Novel methods for delivery of quality healthcare are required to ensure translation of effective, evidencebased fall prevention programmes while containing costs and using limited human resources to maximum effect. ${ }^{13}$ The ultimate success of a health promotion programme depends on its effectiveness and its reach and acceptability in the community. ${ }^{21}$

StandingTall is an engaging balance training programme that is designed specifically for use by older people. It employs technology to deliver an evidence-based, individually tailored exercise programme aimed at improving standing balance and reducing fall risk. The StandingTall programme includes behavioural change techniques to enhance exercise uptake and long-term adherence and is simple for older people to use independently at home. ${ }^{223}$ StandingTall fills an important gap by giving older people the flexibility to exercise unsupervised at home and assists them to meet the required fall prevention exercise dose. While some people enjoy group exercise, some are unable (or unwilling ${ }^{24}$ ) to attend centre-based activities, and many will need to supplement group exercise with home-based exercise to reach their fall prevention target dose. ${ }^{24}$ StandingTall employs technology to provide an effective, long-term method for improving balance and reducing fall risk, using self-management and remote monitoring (adherence, exercise progression, exercise prescription). A large randomised controlled trial in 503 community-dwelling older people has shown that StandingTall is safe, has high adherence rates (median: $90 \mathrm{~min} /$ week over 6 months) and is effective in improving balance and reducing falls in older people. ${ }^{25}$ By combining technology with evidence-based practice, StandingTall will provide a novel solution to the fall epidemic by providing older people with an effective, sustainable, low cost and enjoyable exercise programme thereby supporting older people to remain active and independent. ${ }^{26}$

Understanding factors that may moderate real world uptake and adherence of StandingTall is key to its successful implementation on a broader scale. Process evaluation is a common tool used in pragmatic studies to assess the impact of intervention delivery and access within the study, as well as to gain understanding about how and why the intervention did or did not have its desired impact. The specific aims of this process evaluation are to investigate uptake and adherence to StandingTall by older people; examine how StandingTall is adopted by health professionals; and identify factors that will facilitate the embedding of StandingTall in usual care. While this implementation study was conceived before the 2020 SARS-CoV-2 (COVID-19) pandemic, the delivery of falls prevention services in health and social care systems worldwide has been curtailed because of the need to reduce face-to-face contact. This has given rise to digital delivery of exercise programmes as they offer ways of delivering exercise regimens without, or with reduced, face-to-face contact. ${ }^{27} 28$

\section{METHODS AND ANALYSIS}

This is an international, multisite, pragmatic clinical study with sites in Australia and the UK. The project focuses on implementing the StandingTall programme across three partnering sites in Australia and one site in Northern England, UK. In Australia, two health districts in New South Wales (NSW; Mid-North Coast Local Health District and Northern NSW Local Health District) and one site in Victoria (Austin Health) are involved. In Northern England (UK), participants are recruited from Greater Manchester, North West Coast, Yorkshire and the Humber and the North East. Inherent to the study, is the creation of a website to guide the implementation (www. standingtall.org.au). The website provides information about the StandingTall programme, training modules and resources, with the overall aim to educate older people and their families, as well as exercise therapists and other health professionals, on fall prevention and the StandingTall programme. Website resources include knowledge quizzes with tailored education recommendations, evidence-based fact sheets, safety checklists, StandingTall training modules, manuals, frequently asked questions and blogs.

\section{Participants}

Participants are recruited through health services, community organisations and media advertisements. The study will involve consenting community-dwelling older people, aged 60 years or older with sufficient English language skills to understand study documents. Key exclusion criteria are: residents of aged care facilities; acute medical illnesses, severe psychiatric disorders, progressive neurological diseases including dementia; unstable medical conditions that preclude exercise participation; considered not suitable for study participation by referring family doctor or healthcare worker; have mobility limitations (unable to walk $10 \mathrm{~m}$ indoors without the use of a walking aid) and visual impairment that cannot be corrected with glasses. During the COVID-19 pandemic, 


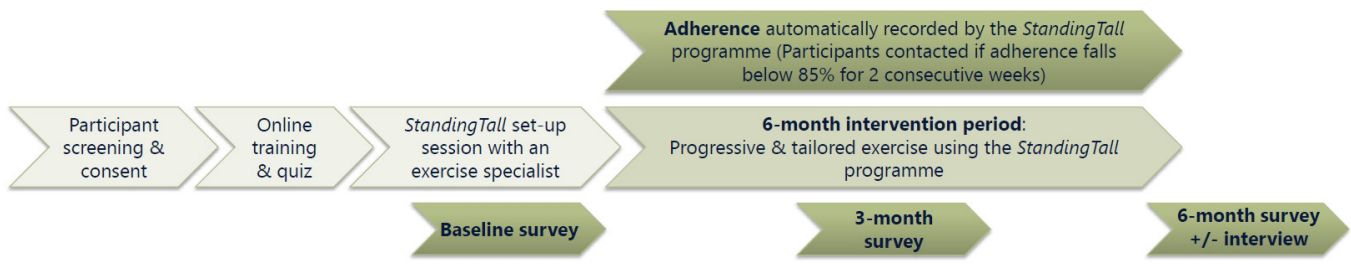

Figure 1 Participants flow through the study.

telehealth set-ups have been introduced which require additional inclusion criteria. Participants are requested to have a support person present who lives in the same house, or is maintaining regular face-to-face contact with the participant, is currently well and can provide supervision and assistance if necessary. Participants who feel that a support person is unnecessary need to be able to step up a street curb without assistance (ie, without using their arms, walking aid or another person) to proceed with the telehealth set-up without a support person. Additionally, in the UK during COVID-19, potential participants who are familiar with strength and balance and known to services could elect to have a telephone setup. Potential participants unfamiliar with strength and balance exercises and who cannot be seen by the exercise specialist/ relevant professionals in person or via telehealth will not be recruited until face-to-face becomes available. Participants are not restricted regarding concomitant care, interventions or activity.

\section{StandingTall intervention programme}

Figure 1 demonstrates participants flow through the study. All participants will be asked to use the StandingTall exercise programme for 6 months. StandingTall comprises standing balance (eg, standing on the floor and/or foam surface), walking (eg, walking in circles or to targets in a grid), stepping (eg, step and lift) and box (eg, step up and over a box) exercises. Exercises are delivered through on-screen animated instructions with video demonstrations and tips. The StandingTall programme delivers unsupervised and progressive balance exercises using digital technology (computer/tablet and internet). The StandingTall programme progresses the weekly exercise dose from $40 \mathrm{~min}$ in the first 2 weeks to 2 hours from week 9 onwards, through fortnightly $20 \mathrm{~min}$ increases. Exercise intensity is individually tailored using a modified perceived exertion 5-point Likert scale (1=unstable, $5=$ very stable). Exercise intensity rating modifies the exercises to ensure they are of moderate-to-high challenge and increases/maintains/decreases exercise intensity according to the user's rating of ability. Participants can choose the timing and duration of their sessions.

Online StandingTall training (including safety) is provided for participants, support persons and exercise specialists through the StandingTall website. The training module includes a safety quiz, where an $80 \%$ pass mark is needed for participants and exercise specialists to receive access to the StandingTall programme and content management system (CMS), respectively. Before commencing the exercise programme, participants are recommended to have a setup session with an exercise specialist (eg, physiotherapist, exercise physiologist, sports scientist, fitness leader or occupational therapist) with prior experience in delivering exercise to older people and who have completed the online training (figure 1). This session can be face-to-face (as intended originally) or via telehealth/video conferencing (during the COVID-19 pandemic and beyond) and is meant to familiarise the participant with the programme and its features, cover exercise safety and complete a balance assessment and a short exercise session. If at any point, the participant, support person or exercise specialist feel that the telehealth/video conferencing set-up is unsafe, the session will be abandoned until such time that additional safety measures can be implemented, or face-toface contact can resume.

The back-end (ie, the CMS) of the StandingTall programme allows research staff and exercise specialists at the local health service level to remotely monitor exercise adherence and progression, as well as adjust exercise categories and intensity (if necessary, eg, in the case of illness or holidays). Participants are contacted by phone if their adherence drops below $85 \%$ for two consecutive weeks to discuss any problems or issues and to encourage adherence (figure 1). If participants need programme support during their 6-month intervention period, they can contact a central study helpline and/or their exercise specialist.

\section{Programme logic model}

The study is conceptualised within a programme logic model, depicted in figure 2, which represents the theory of action or how the intervention contributes to a set of specific outcomes through a series of intermediate results. It is underpinned by a theory of change: Implementation and Knowledge Utilisation Theory. The logic model illustrates how different elements work together to create the desired change in target groups and achieve desired outcomes. Like all models it provides simplifications; the key is for the logic model to show the components of the programme that are thought to be critical for generating outcomes of interest to programme stakeholders. The approach used here is an 'outcomes hierarchy' approach to programme logic. Using this approach, every element of the model is written as an outcome and outcomes are connected. It shows the logical connections between inputs, outputs and outcomes using a type of 'if-then' logic. Figure 2 illustrates the model inputs and how 


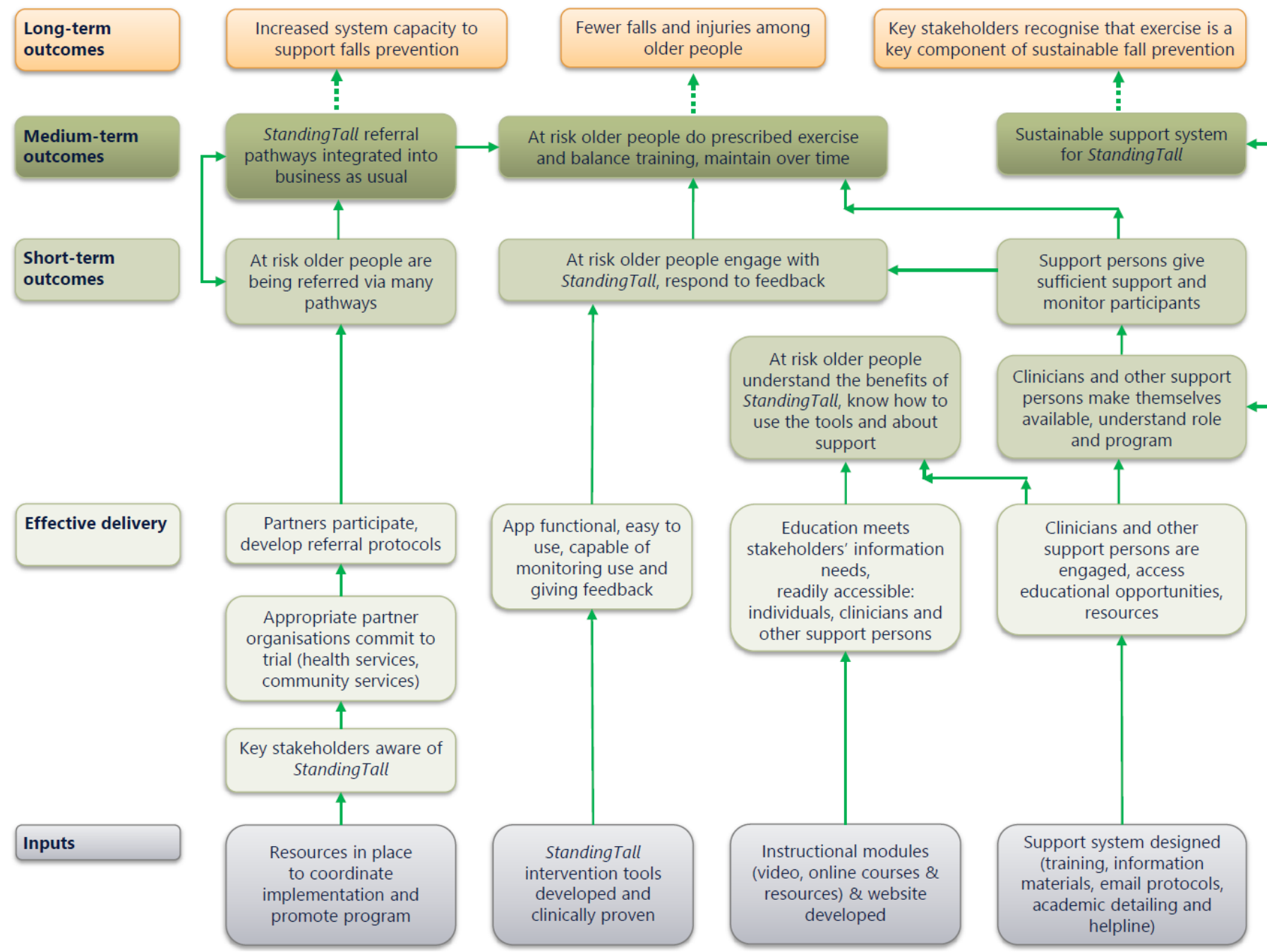

Figure 2 StandingTall Programme logic. (The diagram is read from bottom to top and presents 'if-then' relationships between inputs, effective delivery, and outcomes at different levels).

effective programme delivery will be achieved if these inputs are sufficient.

There are three kinds of outcomes streams for StandingTall-(1) outcomes for participating organisations, which are expected to contribute to increased system capacity to support falls prevention; (2) outcomes for study participants, which are expected to contribute to fewer falls among older people and (3) outcomes for health professionals, which are expected to contribute to more stakeholders recognising that exercise is key to falls prevention (figure 2). Outcomes are categorised as short (3-6 months), medium (12 months) and longer term.

Short-term outcomes relate to adoption, appropriateness, acceptability, fidelity, coverage and feasibility (table 1). Medium and long-term outcomes include fewer falls among older people and increased system capacity to support falls prevention, and relate to sustainability and implementation cost (table 1).

\section{Outcome measures}

The nested process evaluation will use both quantitative and qualitative methods to explore uptake and acceptability of the StandingTall programme to provide direct guidance for programme scale up. Process evaluation data will be collected using StandingTall programme data, study logs and participant, support person, healthcare worker and health service manager surveys and/or interviews (table 1). The data collection time points for participants are presented in figure 1 .

The primary outcome for the study is adherence to the StandingTall programme over 6 months (fidelity; table 1), measured as weekly training dose and total training dose recorded by the programme. The StandingTall programme records actual exercise minutes and does not include rests, watching instructional videos or setup for each exercise. Therefore, a 10 min session could take $20 \mathrm{~min}$ (or sometimes longer) depending on how many instructional videos the user watches and how many rests the user takes. Adherence data are automatically transferred to a secure server at Neuroscience Research Australia. Weekly exercise minutes (adherence) can be monitored in the CMS. 


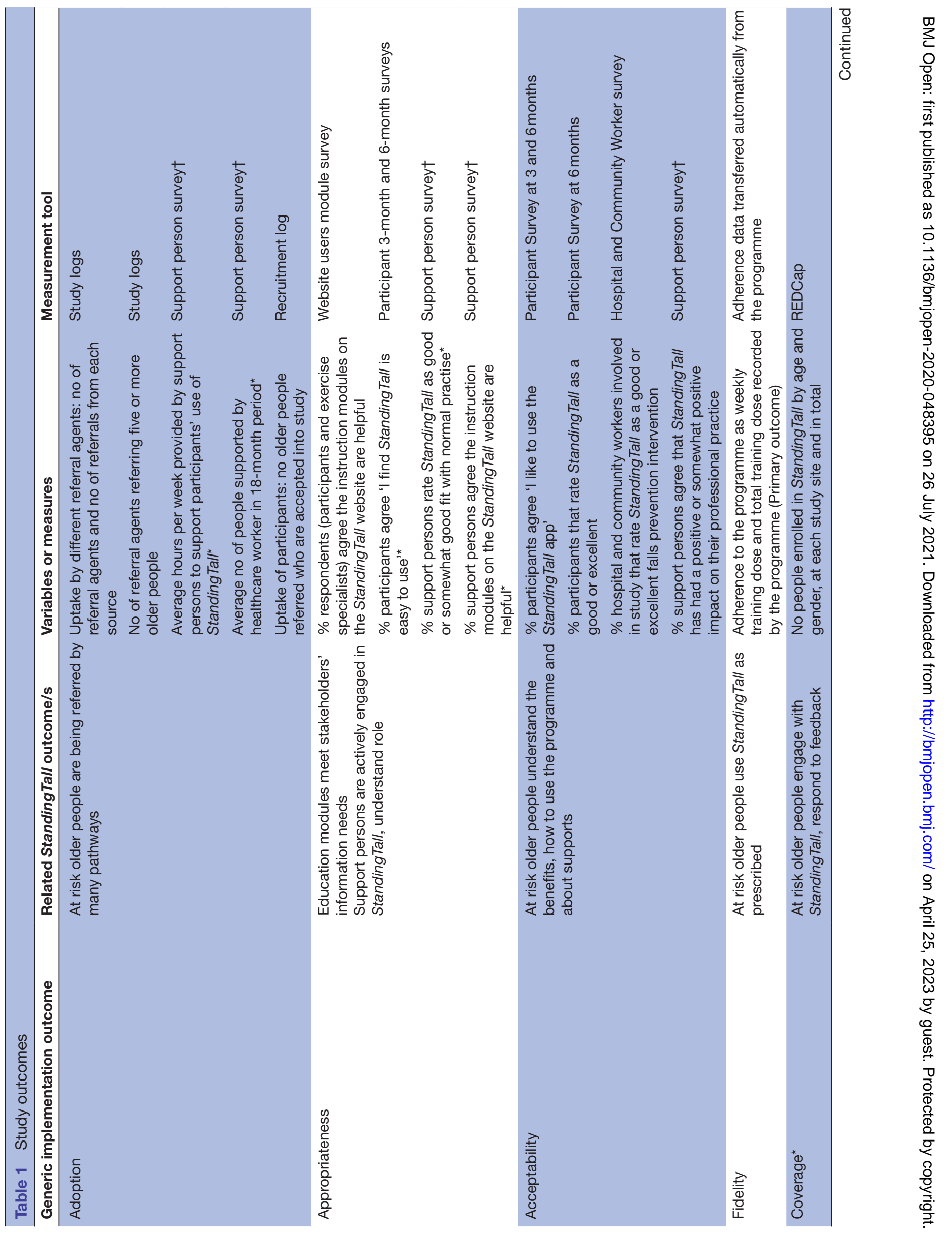




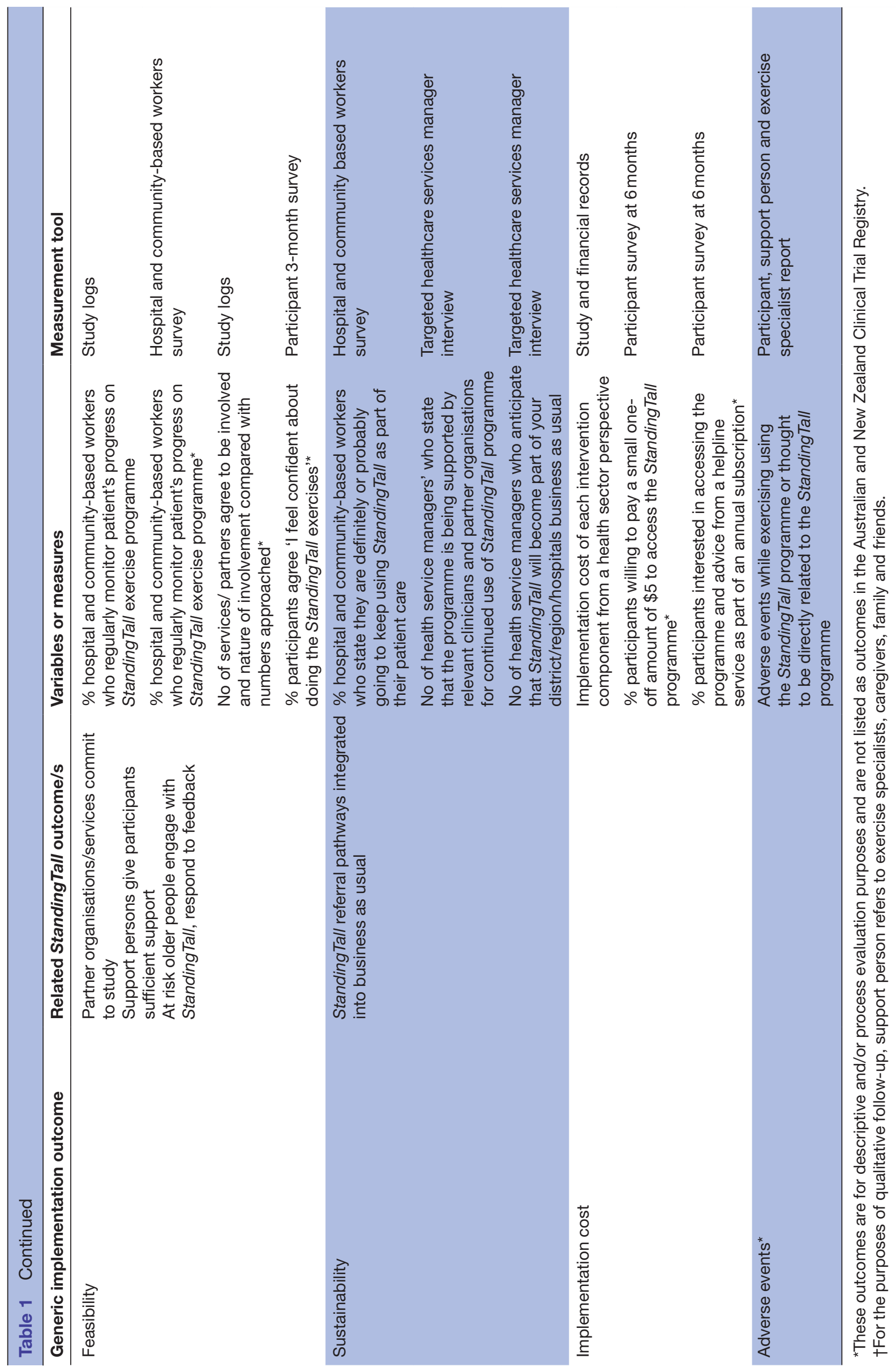


Secondary process evaluation outcomes examine adoption, appropriateness, acceptability, feasibility, sustainability and implementation cost (table 1). The barriers/ facilitators to telehealth/video conferencing delivery (because of COVID-19) will be explored as part of the evaluation.

An adverse event is defined as an unwanted and unusually harmful outcome (eg, exercise-related fall, musculoskeletal injury or cardiovascular event). The event may or may not be related to the StandingTall programme but is recorded if it occurs while the person is participating in the programme (ie, while they are doing StandingTall exercise activities). Adverse events are reported to the data monitoring committee and unexpected and serious adverse events are reported to the governing site and/or Research Ethics Committee (REC), as appropriate and in accordance with the local mandatory reporting policies. Annual reports are provided to the REC and governing sites.

\section{Sample size calculation}

The primary analysis will estimate mean adherence, measured as minutes of exercise per week. With an SD of 43.1 min (estimated from an earlier trial ${ }^{25}$ ), a sample size of 83 participants yield estimates of the mean with a CI width less than or equal to \pm 10 min in $80 \%$ of studies. To allow for an approximately $20 \%$ lost to follow-up, we will recruit 100 participants per site (Australia) or per region (UK).

\section{Statistical analysis}

The nested process evaluation will use both quantitative and qualitative methods to explore uptake and acceptability of the StandingTall programme. Total exercise minutes and mean $(95 \% \mathrm{CI})$ or median (IQR) weekly exercise minutes will be calculated from data recorded by the StandingTall programme and will be reported as adherence (primary outcome). Number and proportions of responses to survey questions will be reported for the secondary outcome measures. Missing data will be left missing; no imputation methods will be used. Interviews will be audiorecorded, transcribed verbatim and thematic analysis will identify patterns within and across study sites. A combination of inductive and deductive coding will be used-commencing with examination of barriers and facilitators, but open to unexpected findings that may contribute to these or other themes. A discrete choice experiment survey will examine the user's preferences and acceptable trade-offs for aspects of StandingTall, including costs and other factors identified by qualitative interviews.

\section{ETHICS AND DISSEMINATION}

Ethical approval and local governance approvals have been obtained (Lead ethics committee in Australia: South Eastern Sydney Local Health District, (HREC 18/288 approved 28/02/2019) and in the UK: North West-Greater
Manchester South Research Ethics Committee (IRAS: 268954 Approved 04/02/2020). All amendment requests will be submitted to these committees. Potential participants will provide verbal consent to be screened for eligibility (figure 1). Written informed consent, or during the COVID-19 pandemic informed online consent, will be obtained from all eligible participants by study staff prior to study enrolment (figure 1). Participant confidentiality and privacy will always be maintained, and all data will be stored securely. Data access will only be provided to study staff and investigators. Health professionals, community referral agents and support persons will be emailed an invitation to complete an online survey. By completing the survey, consent will be implied to participate in the study when they click on the link. If health professionals, community referral agents and support persons are taking part in the interview or focus group sessions, then written informed consent or online informed consent (during the COVID-19 pandemic) will be obtained.

Dissemination will be via publications, conferences, newsletter articles, talks to clinicians and consumers, and meetings with health department and health service mangers. The International Committee of Medical Journal Editors recommended criteria for authorship on publications will be followed. Professional writers will not be used. The full protocol and statistical code will be made available on reasonable request.

\section{Author affiliations}

${ }^{1}$ Falls, Balance and Injury Research Centre, Neuroscience Research Australia, Randwick, New South Wales, Australia

2Prince of Wales Clinical School, Faculty of Medicine, University of New South Wales, Sydney, New South Wales, Australia

${ }^{3}$ School of Health Sciences, University of Manchester, Manchester, UK

${ }^{4}$ Manchester Academic Health Science Centre, Manchester, UK

${ }^{5}$ National Institute for Health Research, Applied Research Collaboration Greater

Manchester, University of Manchester, Manchester, UK

${ }^{6}$ Manchester University NHS Foundation Trust, Manchester, UK

${ }^{7}$ School of Health Sciences, Faculty of Medicine and Health, The University of

Sydney, Sydney, New South Wales, Australia

${ }^{8}$ Centre of Excellence for Population Ageing Research, The University of Sydney, Sydney, New South Wales, Australia

${ }^{9}$ Population Health, Faculty of Medicine, University of New South Wales, Sydney, New South Wales, Australia

${ }^{10}$ The George Institute for Global Health, University of New South Wales, Sydney, New South Wales, Australia

${ }^{11}$ Sydney School of Public Health, Faculty of Medicine and Health, The University of Sydney, Sydney, New South Wales, Australia

${ }^{12}$ Department of Physiotherapy, Austin Health, Heidelberg, Victoria, Australia

${ }^{13}$ Department of Physiotherapy, The University of Melbourne, Melbourne, Victoria, Australia

${ }^{14}$ Health Promotion, Northern NSW Local Health District, Lismore, New South Wales, Australia

${ }^{15}$ ARTD Consultants, Sydney, New South Wales, Australia

${ }^{16}$ Mid North Coast Local Health District, Coffs Harbour, New South Wales, Australia

${ }^{17}$ Physiotherapy, Western Health, St Albans, Victoria, Australia

${ }^{18}$ Australian Institute of Musculoskeletal Science, St Albans, Victoria, Australia

${ }^{19}$ Northern Health Science Alliance, Manchester, UK

Twitter Morag E Taylor @taylor_morag and Chris Todd @Prof_Chris_Todd

Contributors MET, CT, SO, LMC, JC, SRL, TL, DJB, JB, JC, JD, HH-H, WH, KH, PJ, $\mathrm{RL}, \mathrm{GM}, \mathrm{MM}, \mathrm{LM}, \mathrm{CMS}, \mathrm{LW}, \mathrm{NW}, \mathrm{AZ}$ and KD contributed to the design of the study and preparation of the study protocol. KD is chief investigator for the project, SRL, 
$\mathrm{LMC}, \mathrm{JC}, \mathrm{KH}$ and $\mathrm{CT}$ are coinvestigators, MET is project manager in Australia, $\mathrm{HH}-\mathrm{H}$ is Project Manager in the UK and SO is the Australian Project Officer. DJB, AZ, NW CMS, JD are associate investigators from partner organisations. JB, JC, PJ, RL, LM and LW are study staff involved in recruiting, data collection and management, follow-up, technological support and/or app development. KD, WH, MM and MET were involved in planning the evaluation of the study. This manuscript was drafted by authors KD, MET, S0 and WH. MET, CT, SO, LMC, JC, SRL, TL, DJB, JB, JC, JD, HH-H, WH, KH, PJ, RL, GM, MM, LM, CMS, LW, NW, AZ and KD revised the manuscript and all authors approved the submitted manuscript.

Funding This work is supported by a Partnership project grant from the Australian National Health and Medical Research Council (NHMRC; APP 1151934) and funds from New South Wales Ministry of Health. In-kind contributions from New South Wales Ministry of Health, Austin Health, Mid North Coast Local Health District (LHD) Northern New South Wales LHD, the Clinical Excellence Commission, Agency for Clinical Innovation and Uniting also supported this work. SRL and LMC receive salary funding from the NHMRC Fellowship ProgramProgramme. TL is supported by an NHMRC Early Career Fellowship (APP1141392) and the National Heart Foundation Postdoctoral Fellowship (award 101956). In the UK, this research is supported by the National Institute for Health Research (NIHR) Applied Research Collaboration Greater Manchester (NIHR200174), NIHR Senior Investigator Award to CT (NIHR200299), the Northern Health Science Alliance and the NIHR Clinical Research Network Greater Manchester (44434).

Disclaimer The views expressed in this publication are those of the author(s) and not necessarily those of the National Institute for Health Research, the National Health Service, the Department of Health and Social Care nor its partners. The funders have no role in study design; data collection, data management, analyses, interpretation of data, writing the manuscript or the decision to submit the manuscript for publication.

Competing interests WH and MM work for ARTD Consultants who are a consultancy firm paid to evaluate the study.

Patient and public involvement Patients and/or the public were involved in the design, or conduct, or reporting, or dissemination plans of this research. Refer to the Methods section for further details.

Patient consent for publication Not required.

Provenance and peer review Not commissioned; externally peer reviewed.

Open access This is an open access article distributed in accordance with the Creative Commons Attribution Non Commercial (CC BY-NC 4.0) license, which permits others to distribute, remix, adapt, build upon this work non-commercially, and license their derivative works on different terms, provided the original work is properly cited, appropriate credit is given, any changes made indicated, and the use is non-commercial. See: http://creativecommons.org/licenses/by-nc/4.0/.

\section{ORCID iDs}

Morag E Taylor http://orcid.org/0000-0002-4436-862X

Chris Todd http://orcid.org/0000-0001-6645-4505

Lindy M Clemson http://orcid.org/0000-0003-2687-1114

Thomas Lung http://orcid.org/0000-0001-9978-6311

David J Berlowitz http://orcid.org/0000-0003-2543-8722

Helen Hawley-Hague http://orcid.org/0000-0002-2451-4482

Kirsten Howard http://orcid.org/0000-0002-0918-7540

Kim Delbaere http://orcid.org/0000-0002-5655-0234

\section{REFERENCES}

1 Centre for Health Advancement and Centre for Epidemiology and Research. NSW falls prevention baseline survey. NSW department of health. North Sydney: Centre for Health Advancement and Centre for Epidemiology and Research, 2010.

2 Watson W, Clapperton A, Mitchell R. The cost of falls injury among older people in New South Wales 2006/07. NSW Public Health Bulletin 2010;22:55-9.

3 Centre for Health Advancement, Health. NDo Falls - Prevention of Falls and Harm from Falls among Older People: 2011-2015. 30. Sydney: Clinical Excellence Commission, 2011.

4 Moller J. Projected costs of fall related injury to older persons due to demographic change in Australia- revised. Canberra: Commonwealth Department of Health and Ageing, 2003.
5 Gillespie LD, Robertson MC, Gillespie WJ, et al. Interventions for preventing falls in older people living in the community. Cochrane Database Syst Rev 2012;2:CD007146.

6 Sherrington C, Michaleff ZA, Fairhall N, et al. Exercise to prevent falls in older adults: an updated systematic review and meta-analysis. $\mathrm{Br}$ $J$ Sports Med 2017;51:1750-8.

7 Haines TP, Day L, Hill KD, et al. "Better for others than for me": a belief that should shape our efforts to promote participation in falls prevention strategies. Arch Gerontol Geriatr 2014;59:136-44.

8 Kielich K, Mackenzie L, Lovarini M, et al. Urban Australian general practitioners' perceptions of falls risk screening, falls risk assessment, and referral practices for falls prevention: an exploratory cross-sectional survey study. Aust Health Rev 2017;41:111-9.

9 Horne M, Skelton DA, Speed S, et al. Perceived barriers to initiating and maintaining physical activity among South Asian and white British adults in their 605 living in the United Kingdom: a qualitative study. Ethn Health 2013;18:626-45.

10 Simek EM, McPhate L, Haines TP. Adherence to and efficacy of home exercise programs to prevent falls: a systematic review and meta-analysis of the impact of exercise program characteristics. Prev Med 2012;55:262-75.

11 Franco MR, Howard K, Sherrington C, et al. Smallest worthwhile effect of exercise programs to prevent falls among older people: estimates from benefit-harm trade-off and discrete choice methods. Age Ageing 2016;45:806-12.

12 Clemson L. Preventing falls in the elderly: Opportunities and alternatives. In: International Handbook of occupational therapy interventions. Second ed, 2015: 849-57.

13 Lord SR, Sherrington C, Cameron ID, et al. Implementing falls prevention research into policy and practice in Australia: past, present and future. J Safety Res 2011;42:517-20.

14 Healey F, Treml J. Changes in falls prevention policies in hospital in England and Wales. Age Ageing 2013;42:106-9.

15 Cameron K, Schneider E, Childress D. National Council on aging falls Free ${ }^{2}$ National action plan, 2015.

16 Loganathan A, Ng CJ, Tan MP, et al. Barriers faced by healthcare professionals when managing falls in older people in Kuala Lumpur, Malaysia: a qualitative study. BMJ Open 2015;5:e008460-e.

17 Haas R, Haines TP. Twelve month follow up of a falls prevention program in older adults from diverse populations in Australia: a qualitative study. Arch Gerontol Geriatr 2014;58:283-92.

18 Naseri C, McPhail SM, Haines TP, et al. Perspectives of older adults regarding barriers and enablers to engaging in fall prevention activities after hospital discharge. Health Soc Care Community 2020;28:1710-22.

19 Nyman SR, Victor CR. Older people's participation in and engagement with falls prevention interventions in community settings: an augment to the Cochrane systematic review. Age Ageing 2012;41:16-23.

20 McPhate L, Simek EM, Haines TP. Program-related factors are associated with adherence to group exercise interventions for the prevention of falls: a systematic review. J Physiother 2013;59:81-92.

21 Milat AJ, King L, Bauman AE, et al. The concept of scalability: increasing the scale and potential adoption of health promotion interventions into policy and practice. Health Promot Int 2013;28:285-98.

22 Geidl W, Semrau J, Pfeifer K. Health behaviour change theories: contributions to an ICF-based behavioural exercise therapy for individuals with chronic diseases. Disabil Rehabil 2014;36:2091-100.

23 Valenzuela T, Okubo Y, Woodbury A, et al. Adherence to technology-based exercise programs in older adults: a systematic review. J Geriatr Phys Ther 2018;41:49-61. doi:10.1519/ JPT.0000000000000095

24 Yardley L, Donovan-Hall M, Francis K. Attitudes and beliefs that predict older people's intention to undertake strength and balance training. J Gerontol Ser B Psychol Sci Soc Sci 2007;62:P119-25.

25 Delbaere K, Valenzuela T, Lord S. E-Health "StandingTall" balance exercise for fall prevention in older people: results of a two-year randomised controlled trial. BMJ 2021;373:n170.

26 Hawley-Hague $\mathrm{H}$, Boulton E, Hall A, et al. Older adults' perceptions of technologies aimed at falls prevention, detection or monitoring: a systematic review. Int J Med Inform 2014;83:416-26.

27 McGarrigle L, Boulton E, Todd C. Map the apps: a rapid review of digital approaches to support the engagement of older adults in strength and balance exercises. BMC Geriatr 2020;20:483.

28 Doraiswamy S, Abraham A, Mamtani R, et al. Use of telehealth during the COVID-19 pandemic: Scoping review. J Med Internet Res 2020;22:e24087. 